\title{
Applicability of image analysis of neurons and neuroglia of fundus striati using cell profiler software
}

\begin{abstract}
Analysis of volumetric and morphological neuronal data has been of keen interest to neurologists and neuroscientists because of its implications in pathological conditions such as schizophrenia, autism, obsessive compulsive disorder etc. One such part of human brain which has been explored in recent years is fundus striati, a part of basal ganglia bridging caudate nucleus and putamen. A versatile technique to measure neurons of fundus striati is the use of Cell profiler software. The present study was undertaken to study and analyze images of fundus striati using cell profiler as an automated image analysis technique. A qualitative cross sectional study was done using eighty one images of histological sections of hematoxylin and eosin stained $8 \mu$ thick tissue sections of fundus striati. Freely downloadable Cell Profiler software was installed, specific pipeline for neuronal assay was drawn, and modules were used to analyze images. Neuronal nuclei were identified as primary objects and cytoplasm was identified by the software as secondary objects. Using specific modules, neurons and branching points of their neurites in images of fundus striati were measured in pixel units. Mean Intensity units of neurons of fundus striati as measured by intensity module of Cell profiler was 17.916 pixel units. Results of the present study can be extrapolated to correlate with pathological conditions associated with emotional and behavioral disorders involving fundus striati. Cell profiler is cost effective software which is beneficial to identify and measure neurons of fundus striati.
\end{abstract}

Keywords: fundus striati, cell profiler, image analysis
Volume 5 Issue 4 - 2018

\author{
Vasudha Kulkarni, Ramesh BR \\ Department of Anatomy, DR. B.R.Ambedkar Medical College, \\ India
}

Correspondence: Vasudha Kulkarni, Associate Professor Department of Anatomy, Kadugondanahalli, DR. B.R.Ambedkar Medical College, Bangalore, India, Tel 9008825690 , Emailvasu_anil77@rediffmail.com

Received: April 0I, 2018 | Published: July 18, 2018

\section{Introduction}

Constant changing patterns of activity of billions of neurons have been extensively studied by thousands of scientists and neurologists as a matter of scientific rigor and most importantly for updating disease prevention and control measures.

The cellular composition of human nervous tissue comprises of three basic cell types: neurons, neuroglia and endothelial cells. The number, density, volume and morphology of neurons and neuroglia correlate with changes with brain function. ${ }^{2}$ Caudate nucleus and Globus pallidus of Lentiform nucleus together are termed as Corpus striatum. The island of striatum caudal to anterior limb of internal capsule which is a connecting bridge between caudate nucleus and globus pallidus is termed as Fundus striati. It comprises of small and large sized neurons and splintering fibre bundles. ${ }^{3}$ One of indigenous part of fundus striati is nucleus accumbens. This area has island of small sized neurons with dopaminergic 3 receptors. This area has been extensively studied for interaction of antipsychotic drugs and drug abuse due to its conundrum with amygdale. ${ }^{4}$

Identification of deep brain structures such as basal ganglia and their histological evaluation is also imperative for deep brain surgeries, treatment of tremor, and precise localization of nuclei in non-motor disease such as obsessive compulsive disorder etc. ${ }^{5}$ Schizophrenic patients have lower brain volume in cortical regions due to reduced number of neuroglia. Autistic people have additional synapses and more branches of neurons. ${ }^{2,5}$ Various methods are available for quantitative microscopic analysis of nervous tissue such as plot/ quadrant sampling, fractionator method, stereological method, flow cytometry method, isotropic fractionator etc. These methods have their limitations such as availability of virtual imaging software which is expensive, error of judgment and high inter observer variability. ${ }^{6-10}$ The present study has used one such software called as Cell Profiler to overcome these limitations. The advantage of Cell Profiler over these methods is its applicability to thousands of images as an automated biological image analysis package. It can be customized and tailored to needs of individual studies.

Cell Profiler software is freely downloadable software available from website http://cellprofiler.org/. There is a manual available in the website which has technical description of algorithms and technical description. A series of pipelines are available in downloadable format which consists of modules for image analysis steps. ${ }^{11}$ In tissues, cells are adherent to one another which makes analysis nearly impossible. Cell profiler recognizes and separates the clumped cells, dividing lines drawn between cells and similar cells are removed or merged together based on their size or shape. Cell Profiler identifies and delineates (or "segments") nucleus and cell body, and extracts a number of measurements from each cell. Using the point-and-click graphical user interface (GUI), users construct an image analysis "pipeline", a sequential series of modules that each performs an image processing function such as illumination correction, object identification (segmentation), and object measurement. Modules can be mixed and matched and their settings can be adjusted to measure the phenotype of interest. The present study was done to provide a user friendly interface of cost effective and faster image analysis of fundus striati of basal ganglia using Cell Profiler software. ${ }^{12}$ The objectives of the present study were to identify and analyze neurons of fundus striati. 


\section{Methods}

Ethical clearance was obtained prior to conduct of study from institutional ethical committee. A total of 81 images with approximately 68 neurons per image were automated for process of identifying and measuring neurons.

Inclusion and exclusion criteria: There are many freely accessible software which make biological imaging cost effective such as Image J, Java image processing and analysis program by NIH Fiji, a distribution of Image J, TIMWIN downloadable from Garbo, SIGMASCAN downloadable from SYSTAT, Image Pro Plus downloadable from Media Cybernetics, DA Cell Counter from Yamato. All these software have their applications in various scientific fields such as astronomy, ecology and medicine. Cell Profiler project is also one such software which is based at the Broad Institute Imaging Platform. ${ }^{11,13,14}$ This software is freely downloadable and image analysis is exclusively of biological tissues. Since it can process bio formats easily, analysis of nervous tissue would be easier, hence this software was chosen.

Coronal section of formalized human cadaveric brain was done at the level of anterior perforated substance..$^{15} 1.5$ cubic centimeter section of fundus striati was dissected, processed, paraffin embedded and 8 micrometer thick sections were made using microtome. These tissue sections were brought to water, stained with hematoxylin and eosin stain and mounted with DPX. The photographs of fundus striati were obtained using Labomed microscope under $40 \mathrm{x}$ objectives and saved in PNG (Portable Network Graphics) format (Figure 1).

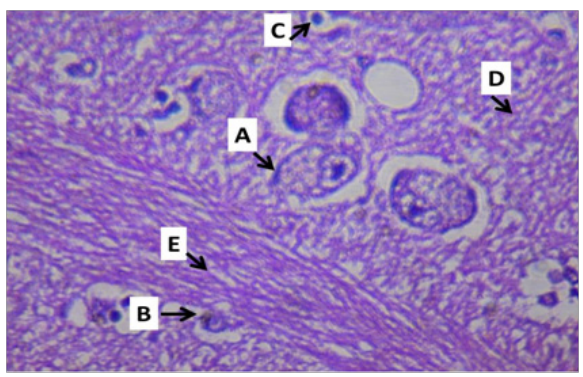

Abbreviations: A, large neurons of caudate nucleus; B, small neurons of putamen; C, neuroglia with small hyperchromatic nucleus; D, Neurites; E, anterior limb of internal capsule.

Figure I Fundus striati section-8 $\mu$ thick, hematoxylin and eosin stain, $40 \mathrm{X}$ magnification.

Freely available Cell profiler image analysis software was downloaded from http://cellprofiler.org. Java 8 is a prerequisite and whether windows support 32 bit or 64 bit software has to be checked before download: ${ }^{16}$

a. The images to be analyzed were taken from fundus striati tissue of human brain specimen. A text file called "Fundus striati.csv" was created to save information about 81 images.

b. The first task was to set up a Cell Profiler pipeline consisting of a number of individual modules; this is a prerequisite because each module performs a unique image-processing step, and multiple modules can be arranged such that they are processed in sequential order. ${ }^{11}$

c. The next task was to test pipeline on a few images so that we had optimized in the settings. Once optimization was complete, we ran the pipeline on all the images of fundus striati, collected measurements from each image and stored them in a database. d. The last task was to use Cell Profiler software for identifying features and obtaining measurements from cellular images.

\section{Starting cell profiler and configuring the input data for analysis}

Downloaded Cell Profiler software was started from the Start Menu (Windows) (Figure 2). In the Cell Profiler interface, on the left hand side of panel, input data consisted of four crucial components namely

a. Images(This is to drag and drop images to be analysed)

b. Metadata (This module provides information about the neurons in each image)

c. Names and Types (This is the third module in the Input module panel which allows to assign a name to each image by which other modules will refer to it.)

d. Group For the "Assign a name to" setting, select "Images matching rules" from the drop-down list to identify images. ${ }^{11}$ Figure 2 depicts display window of cell profiler software showing input modules and analysis modules on left side and module notes and module settings on right side.

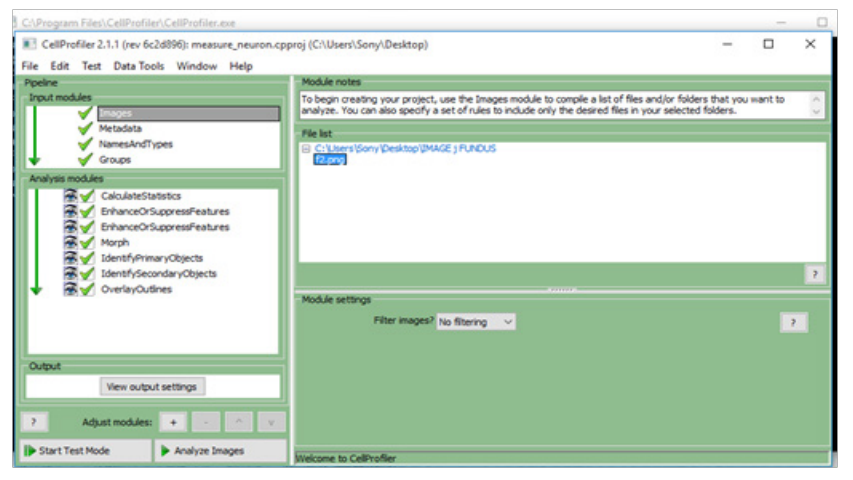

Figure 2 Display window of cell profiler showing input modules.

\section{Results}

\section{Building a pipeline}

The software uses the concept of a pipeline, a series of modules (Figure 3). Each module performs a specific task on the image or on identified objects. ${ }^{11,16} \mathrm{We}$ downloaded example pipeline from website http://forum.cellprofiler.org/uploads/default/original/2X/f/ f871606d28757a40da3ccd01900b485d0f284a50.cpproj

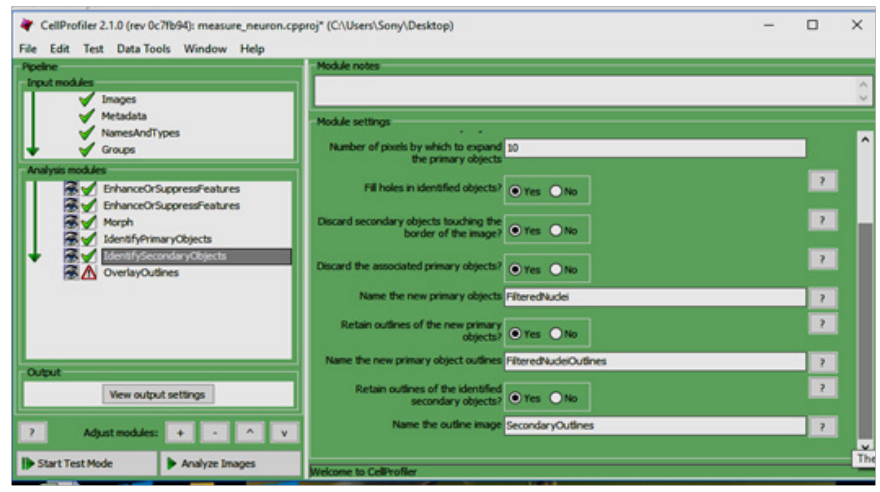

Figure $3 \mathrm{~A}$ pipeline of modules used for analysis of neurons in fundus striati. 
This pipeline consisted of these features:

a. Enhance Or Suppress Features - used to enhance the neurites,

b. Apply Threshold - used to enhance the cell body,

c. Identify Primary Objects - identifies the nucleus,

d. Identify secondary Objects- - identifies the cell body,

e. Apply Threshold - to create a binary image,

f. Morph - used the skeletal module to generate skeletonized image,

g. Measure Neurons,

h. Export to Spreadsheet

In nutshell, using this pipeline, images of fundus striati were processed for highlighting neurites, identifying neurons and their nuclei and measuring neurons (Figure 3).

\section{Application of pipeline features of specific analysis modules}

\section{Module}

Enhance Or Suppress Features enhances or suppresses certain image features (such as speckles, ring shapes, and neurites). This module enhances or suppresses the intensity of certain pixels relative to the rest of the image, by applying image processing filters to the image. A grayscale image was produced in which objects would be identified using an Identify module. This was a prerequisite for subsequent identification of objects. ${ }^{16}$ Figure 4 depicts original image of fundus striati on the left side and enhanced features of fundus striati on right side. Figure 5 shows further enhanced of membrane features of neurites of fundus striati.

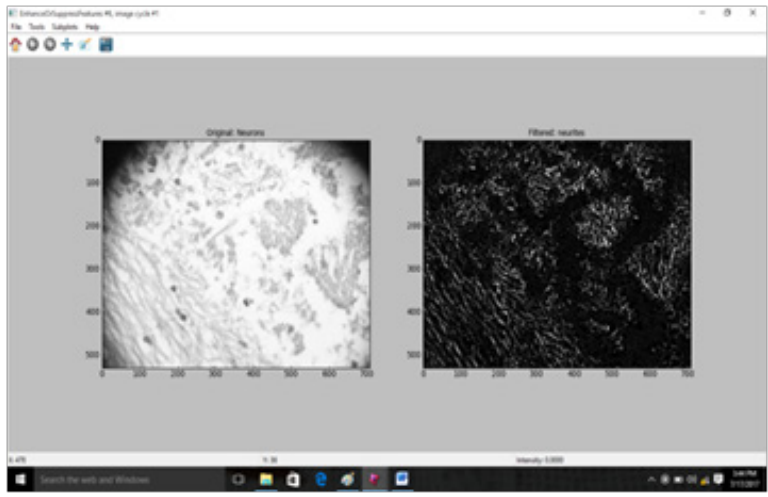

Figure 4 Enhance neurites.

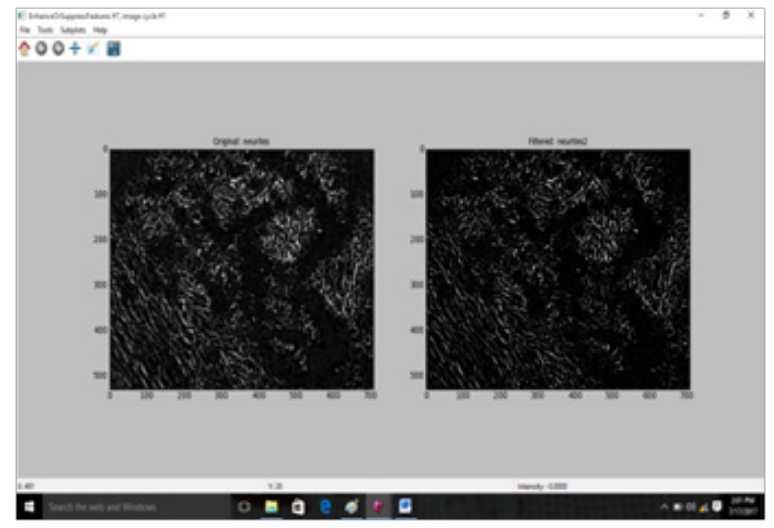

Figure 5 Enhance neurites.

\section{Morph neurons}

This module performed low-level morphological operations on binary or grayscale images, resulting in an image of the same type. ${ }^{16}$ This algorithm minimized the interference of masked pixels; for instance, the dilate operation will only consider unmasked pixels in the neighborhood of a pixel to the first neighborhood pixel in the structuring element. ${ }^{11}$ Here , nucleus is considered as circular element and Morph feature brings this element into forground and neighbouring elements/ pixels are sent to background. Morph feature is a prerequisite for measuring neurons (Figure 6).

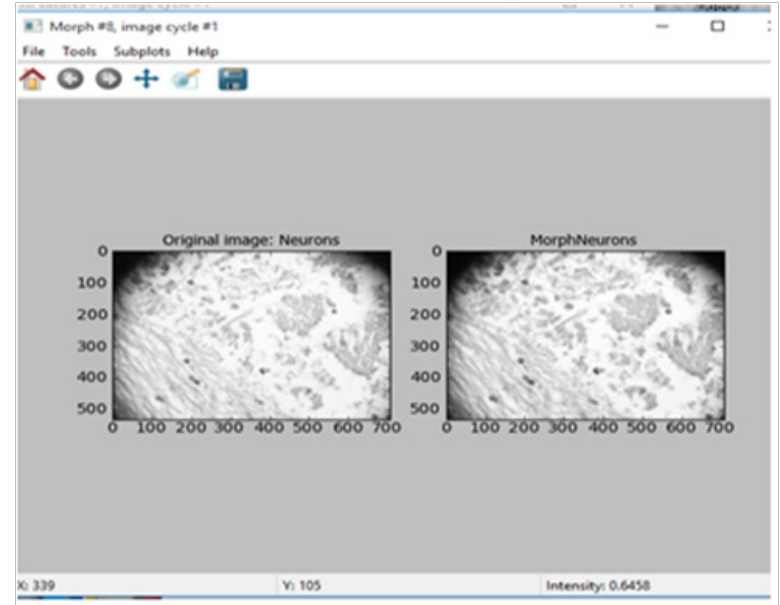

Figure 6 Module showing morphological operations on grayscale image.

\section{Module apply threshold}

This application is needed to set pixel intensities below or above a certain threshold to zero. The result is either a grayscale or binary image based on a threshold which can be preselected or calculated automatically using one of many methods. The intensity threshold affects the decision of whether each pixel will be considered foreground (region(s) of interest) or background. ${ }^{16}$ A higher threshold value will result in only the brightest regions being identified, whereas a lower threshold value will include dim regions (Figure 7). We can have the threshold automatically calculated from a choice of several methods, or we can enter a number manually between 0 and 1 for the threshold. ${ }^{11}$

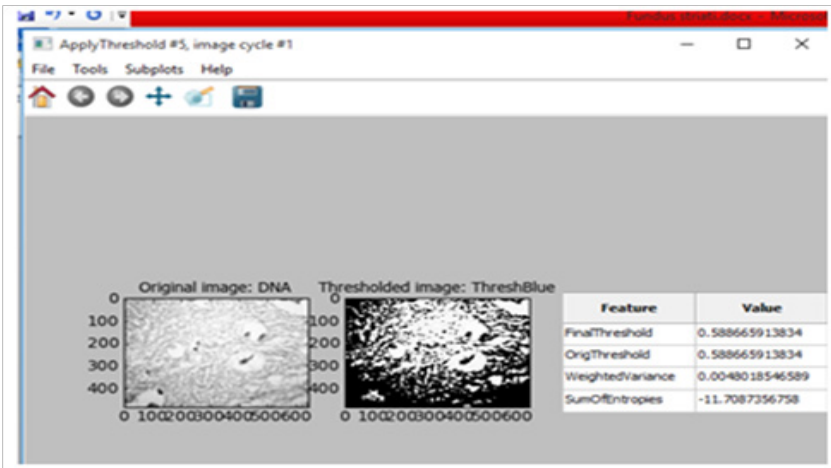

Figure 7 Module showing results of application of threshold for grayscale image of fundus striati. 


\section{Identifying the nuclei as the "primary objects" (Figure 8)}

The goal is to have the outlines match the actual nuclei boundaries precisly, as well as separating touching objects accurately. ${ }^{16}$ In other words, if we want the program to split a single object (in this case, a single neuron) into multiple objects, and we do want to merge multiple objects into a single object. ${ }^{11}$

The results of Module, Identify Primary Objects Figure 8 are displayed in four panels in the display window, as shown in Figure 6: A-The raw image, titled as "Original image" plus the image number
B-Colored image of the identified and labeled objects, titled with the object name (in this case, "Nuclei"). Here colors themselves are arbitrary, intended to distinguish each identified object from its neighbors. C- An image of the object outlines superimposed on the raw image, titled with the object name. Green outlines around an object indicate that the object has passed the selection criteria in the module. Yellow outlines indicate that the object touches the image edge, and has therefore been excluded. Red outlines indicate objects that do not pass a size criterion, and have therefore been excluded. D- Table of module setting values and statistics. ${ }^{11,16}$

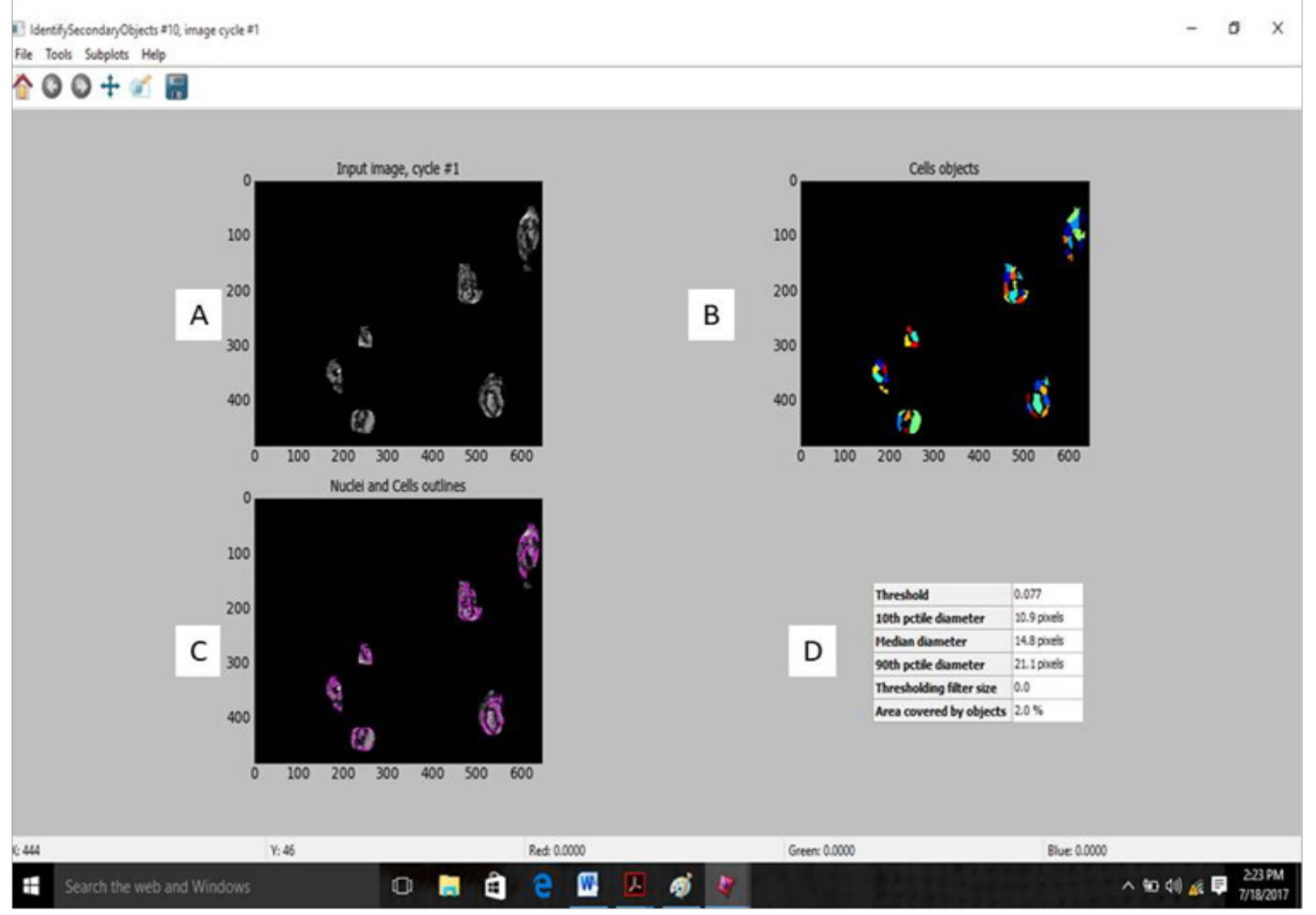

Figure 8 Module showing results of identifying primary objects.

\section{Identify secondary objects}

In Cell Profiler, if nucleus is identified as primary object, cytoplasm is recognised as secondary object. So it is mandatory to execute module of identifying primary object first followed by secondary object. For densely-packed cells, determining the cell borders using a cell body stain can be quite difficult since they often have irregular intensity patterns and are lower contrast with more diffuse staining. In addition, cells often touch their neighbors making it harder to delineate the cell borders16. It is often easier to identify an organelle which is well separated spatially (such as the nucleus) as an object first and then use that object to guide the detection of the cell borders. In order to identify the edges of secondary objects, this module performs two tasks: Finds the dividing lines between secondary objects which touch each other and finds the dividing lines between the secondary objects and the background of the image (Figure 9). In most cases, this is done by thresholding the image stained for the secondary objects. ${ }^{11}$

The fact to be considered that the primary object will always be completely contained within a secondary object. Here the nucleus of neurons and neuroglia are completely enclosed within identified cells. $^{12}$

\section{Module: measure object intensity}

It measures several intensity features for identified neurons. Given an image with objects identified (e.g. nuclei or cells), this module extracts intensity features for each object based on one or more corresponding grayscale images (Figure 8). Measurements were recorded for each neuron in pixel units. Intensity measurements were made for all combinations of the images and objects entered. If we want only specific image/object measurements, we can use multiple Measure Object Intensity modules for each group of measurements 
desired. ${ }^{16}$ The units of intensity from microscopy images are usually described as "Intensity units" or "Arbitrary intensity units" since microscopes are not calibrated to an absolute scale (Figure 10). Also, it is important to note whether we are reporting either the mean or the integrated intensity, so we must specify "Mean intensity units" or "Integrated intensity units" accordingly. ${ }^{11}$

\section{Measure neurons}

This module is helpful for measuring branching information for neurons or any skeleton objects with seed points (Figure 11). The module measures the number of trunks and branches for each neuron in an image. The module takes a skeletonized image of the neuron plus previously identified seed objects (for instance, the neuronal soma) and finds the number of axon or dendrite trunks that emerge from the soma and the number of branches along the axons and dendrites. ${ }^{16}$ It is to be noted that the seed objects must be both smaller than, and touching the skeleton in order to be counted. ${ }^{11}$

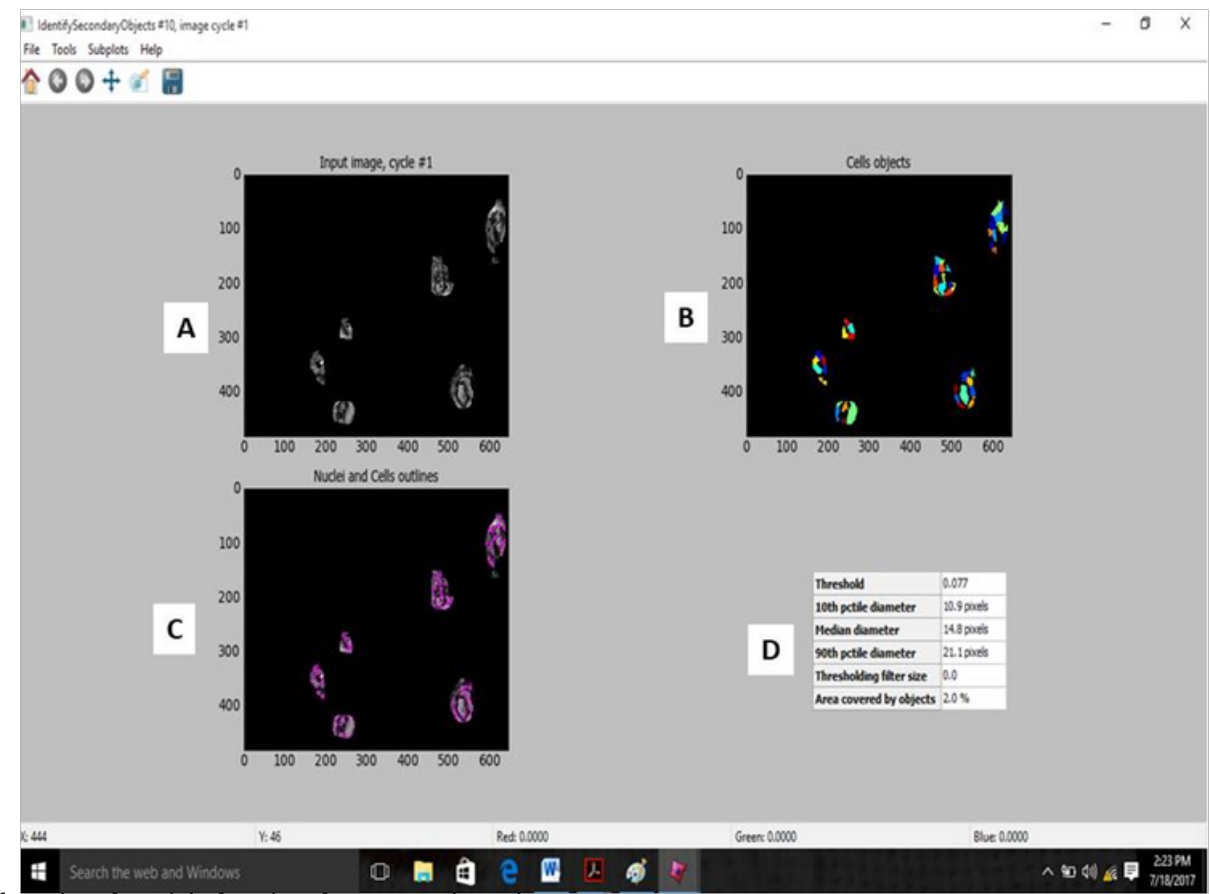

Figure 9 Display of results of module for identifying secondary objects.

\begin{tabular}{|l|l|l|l|l|l}
\multicolumn{1}{|c|}{ Image } & Object & \multicolumn{1}{c|}{ Feature } & \multicolumn{1}{c|}{ Mean } & Median & \multicolumn{1}{c}{ STD } \\
\hline FilteredBlue & Cells & IntegratedIntensity & 17.916 & 14.51 & 13.67 \\
\hline FilteredBlue & Cells & MeanIntensity & 0.089 & 0.089 & 0.017 \\
\hline FilteredBlue & Cells & StdIntensity & 0.027 & 0.025 & 0.01 \\
\hline FilteredBlue & Cells & MinIntensity & 0.028 & 0.033 & 0.015 \\
\hline FilteredBlue & Cells & MaxIntensity & 0.159 & 0.155 & 0.035 \\
\hline FilteredBlue & Cells & IntegratedIntensityEdge & 5.83 & 5.367 & 2.081 \\
\hline FilteredBlue & Cells & MeanIntensityEdge & 0.08 & 0.077 & 0.015 \\
\hline FilteredBlue & Cells & StdIntensityEdge & 0.026 & 0.024 & 0.011 \\
\hline FilteredBlue & Cells & MinIntensityEdge & 0.03 & 0.035 & 0.015 \\
\hline FilteredBlue & Cells & MaxIntensityEdge & 0.147 & 0.141 & 0.037 \\
\hline FilteredBlue & Cells & MassDisplacement & 0.476 & 0.384 & 0.363 \\
\hline FilteredBlue & Cells & LowerQuartileIntensity & 0.07 & 0.071 & 0.013 \\
\hline FilteredBlue & Cells & MedianIntensity & 0.089 & 0.09 & 0.018 \\
\hline FilteredBlue & Cells & MADIntensity & 0.019 & 0.018 & 0.008 \\
\hline FilteredBlue & Cells & UpperQuartileIntensity & 0.109 & 0.108 & 0.024 \\
\hline FilteredBlue & Cells & CenterMassIntensity_X & 430.96 & 483.337 & 157.574 \\
\hline FilteredBlue & Cells & CenterMassIntensity_Y & 269.246 & 279.819 & 124.126 \\
\hline FilteredBlue & Cells & MaxIntensity_X & 430.783 & 484.5 & 157.72 \\
\hline FilteredBlue & Cells & MaxIntensity_Y & 268.0 & 280.0 & 123.613 \\
\hline
\end{tabular}

Figure 10 Module showing results of measuring object intensity. 


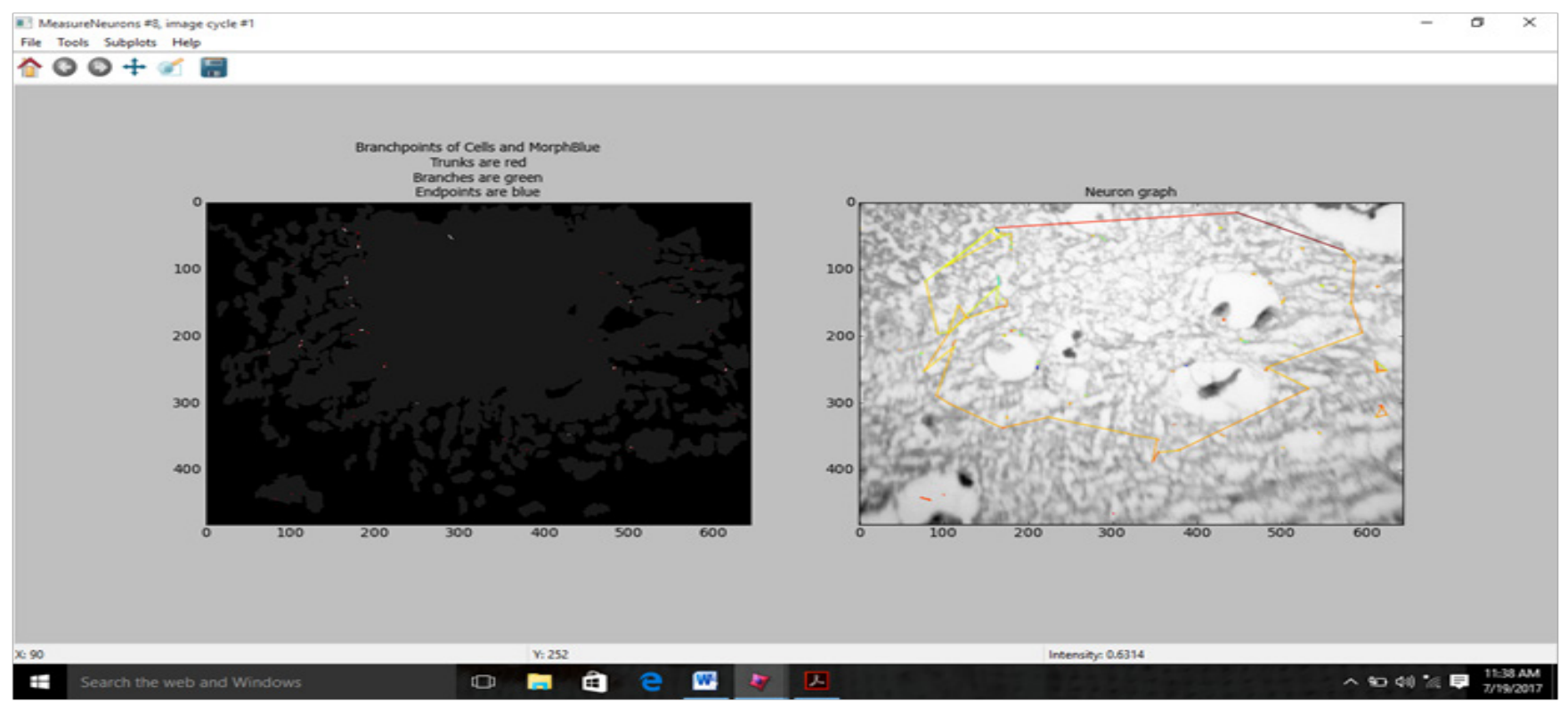

Figure I I Module showing results of measuring neurons.

\section{Discussion}

The present study used Cell Profiler as a flexible platform for identifying neurons, determining their number and records its measurements. This is an effort to produce rapid, cost effective and quantitative results of components (specifically neurons) in fundus striati so that a wide arena of information about its biological mechanisms can be extrapolated. In comparison to other software, the present study yielded statistically significant results of measurements of neurons of fundus striati of cadaveric human brains.

Studies have shown that fundus striati is involved in motivational regulation of instrumental performance and expression of responding in Pavlovian conditioning. ${ }^{17}$ Further, when the addictive drug, cocaine, was repeatedly given to rats and then withdrawn, a subsequent challenge with this drug induced enhanced cFos and JunB expression in striosomes and some ventral striatal regions relative to the pattern seen in acutely treated rats. ${ }^{18,19}$ The fundus striati (also called the amygdalo-striatal transitional zone) also contained a dense Calcitonin gene related Peptide network and terminals without basket-type terminals. This uneven distribution of CGRP within the caudate putamen has been proven to be very peculiar; cholecystokinin and TIP39 are the only other neuropeptides known to have a similarly uneven striatal distribution. ${ }^{20,21}$

Volume data of neurons has enormous clinical relevance in the fields of neuropsychiatry and neuroimaging. Earlier studies have shown that neuronal number in right basal forebrain is higher than its left counterpart. ${ }^{21}$ Certain antipsychotic drugs such as olanzapine and haloperidol have shown to reduce brain weight and volume in macaque monkeys. ${ }^{2}$ The present study has implications in the field of pharmaceutical screening market as software can give information about neurite growth, micronucleus formation, protein translocation. ${ }^{22}$ Cell Profiler can extract valuable biological information from images quickly while increasing the objectivity and statistical power of assays. It helps researchers approach a variety of biological questions quantitatively, including standard assays (e.g., cell count, size, per-cell protein levels) as well as complex morphological assays (e.g., cell/ organelle shape or subcellular patterns of DNA or protein staining). ${ }^{20}$

\section{Advantages of cell profiler analysis of fundus striati}

Human microscopic observation of fundus striati is qualitative, we can recognize whether it is neuron or neuroglia. This is because of the fact that neuronal nuclei are euchromatic with prominent nucleolus whereas neuroglia is heterochromatic. We have to scroll through entire optical field patiently and cautiously to count the number of neurons and identify any abnormal branching pattern of neurites. On the contrary, Cell profiler helps in quantitative analysis, could group similar cellular features of neurons together. ${ }^{16}$ Earlier study done to construct a three dimensional atlas of basal ganglia based on Immunohistochemical and Magnetic Resonance Imaging data has proven that for histological level of resolution of images 3D coherency is needed5. So, a high degree of resolution is mandatory for such procedures unlike Cell profiler analysis which can cater the needs of histological images using specific modules easily.

\section{Key challenges faced during the study}

Installation and configuration of software was a herculean task. Since the software package is Java based, unless Java is installed and run images can't be processed. Cell Profiler software runs on concept of pipeline (a set of specifications to frame a module) which should be predetermined. Images ideally must be PNG (Portable Network Graphics) or TIF (Tag Image File) format. JPG or JPEG format of images are "lossy" (information is lost in the conversion to the format) and cannot be analyzed by Cell Profiler. Every step in module must be cautiously determined and followed lest we would not get the expected results.

\section{Future ahead}

This project is the initial step to explore possibilities and application of cell profiler software to analyze fundus striati of basal ganglia. There is a need for volumetric and morphometric analysis of neurons and neuroglia of fundus striati. So, as a next step, serial sections of entire tissue block of fundus striati shall be processed, stained and analyzed using cell profiler software to measure volume of neurons(both small and large sized), number of neurons and volume of neuroglia. Further using immunohistochemistry technique, images of fundus striati shall be processed for image analysis to explore its functional and statistical 
correlation in normal and pathological situations.

\section{Conclusion}

Fundus striati, an area of brain has been extensively explored to know its role in addictive behavior of individuals. The present study attempted to apply Cell Profiler software to study and analyze images of neurons of fundus striati. Cell Profiler software helped in identifying neurons of fundus striati, and measuring branching pattern of its neurites. This software gains advantage over other image analysis software in being simpler, easily accessible with readily available pipeline modules in a cost effective so that images of neurons of fundus striati could be measured with statistical accuracy. As a next step, the research will be further taken up to explore one of the areas of fundus striati: nucleus accumbens and extract its volumetric data by compiling modules of Cell profiler software as automated image software.

\section{Acknowledgements}

Our sincere thanks to Dr. Poonam DN and Dr. Shylaja DK, Assistant Professors, Department of Anatomy, DR. B. R. Ambedkar Medical College, Bengaluru, Dr. Balachandra N, Professor and HOD, Department of Anatomy, East Point Medical College for their valuable inputs for write up of this project. We extend our special thanks to Mrs. Reeja, technician, DR. B. R. Ambedkar Medical College for tissue processing and staining. We extend our thanks to cell profiler software analysts who have provided us with platform for neuronal analysis.

\section{Conflict of interest}

Author declares that there is no conflict of interest.

\section{References}

1. Susan Standring. Neuroanatomy. In: Alan A Crossman. Gray's Anatomy the Anatomical Basis of Clinical Practice. Spain: Elsevier publisher; 39th ed. 2008:225.

2. Agata Kolodziejzyk, Magdalena Ladniak, Adam Piorkowski. Constructing software for analysis of neuron, glial and endothelial cell numbers and density in histological cell numbers and density in histological nissl-stained rodent brain tissue. Journal of Medical Informatics \& Technologies. 2014;23:77-86.

3. Harold Brockhaus. The finer anatomy of the septum and of the striatum with 72 illustrations. NIH Library Translation (NIH-81-109). J Psychol Neurol. 1942;51(1-2):1-56.

4. Lennart Heimer, Gary W Van Hoesen, Michael Trimble, et al. The ventral striatopallidal system. In: Anatomy of neuropsychiatry the new anatomy of the basal forebrain and its implications for neuropsychiatric illness. Netherlands: Elsevier Inc; 2008:77-82.

5. Yelnik J, Bardinet E, Dormont D, et al. A three-dimensional, histological and deformable atlas of the human basal ganglia. I. Atlas construction based on immunohistochemical and MRI data. Neuroimage. 2007;34(2):618-638.

6. Carlezon WA Jr, Thomas MJ. Biological substrates of reward and aversion: A nucleus accumbens activity hypothesis. Neuropharmacology. 2009;56 Suppl 1:122-132.

7. Young NA, Flaherty DK, Airey DC, et al. Use of flow cytometry for highthroughput cell population estimates in brain tissue. Front Neuroanat. 2012;6(27):1-8.

8. Schmitz C, Hof PR. Recommendations for straightforward and rigorous methods of counting neurons based on a computer simulation approach. J Chem Neuroanat. 2000;20(1):93-114.

9. Weidi Xie, Alison Noble J, Andrew Zisserman. Microscopy cell counting with fully convolutional regression networks. 2017:1-8.

10. Armstrong RA. Quantitative microscopic analysis of histological sections of brain tissue. Modern Research and Educational Topics in Microscopy. 2007:442-452.

11. Cell profiler.

12. Jones TR, Kang IH, Wheeler DB, et al. Cell Profiler Analyst: data exploration and analysis software for complex image-based screens. BMC Bioinformatics. 2008;9:482.

13. French AP, Wilson MH, Kenobi K, et al. Identifying biological landmarks using a novel cell measuring image analysis tool: Cell-o-Tape. Plant Methods. 2012;8:7.

14. Carpenter AE, Jones TR, Lamprecht MR, et al. Cell profiler: image analysis software for identifying and quantifying cell phenotypes. Genome Biol. 2006;7(10):R100.

15. Romanes GJ. Cunningham's manual of practical anatomy: head and neck and brain. 15th ed. India: Thomas press; 2007;3:289.

16. Cell profiler cell image analysis software manual.

17. Basar K, Sesia T, Groenewegen H, et al. Nucleus accumbens and impulsivity. Prog Neurobiol. 2010;92(4):533-557.

18. Léna I, Bradshaw S, Pintar J, et al. Adaptive changes in the expression of central opioid receptors in mice lacking the dopamine $\mathrm{D} 2$ receptor gene. Neuroscience. 2008;153(3):773-788.

19. Dobolyi A, Irwin S, Makara G, et al. Calcitonin gene-related peptidecontaining pathways in the rat forebrain. J Comp Neurol. 2005;489(1):92119

20. Tempel A, Zukin RS. Neuroanatomical patterns of the mu, delta, and kappa opioid receptors of rat brain as determined by quantitative in vitro autoradiography. Proc Natl Acad Sci. 1987;84(12):4308-4312.

21. Liu FC, Graybiel AM. Region-dependent dynamics of cAMP response element-binding protein phosphorylation in the basal ganglia. Proc Natl Acad Sci. 1998;95(8):4708-4713.

22. Carolina Wählby, Martin Simonson, Megan Rokop, et al. Image-based screening using subcellular localization of FOXO1A in osteosarcoma cells: a computer exercise using cell profiler \& cell profiler analyst software. 2017. 